\title{
Noll's axioms and formulation of the closure relations for the subgrid turbulent tensor in Large Eddy Simulation
}

\author{
FRANCESCO GALLERANO, GIOVANNI CANNATA \\ Department of Civil, Constructional and Environmental Engineering \\ "Sapienza" University of Rome \\ Via Eudossiana 18, 00184
}

ITALY

\begin{abstract}
In this paper, the relation between the Noll fo rmulation of th e principle of material frame indifference and the principle of turbulent frame indifference in large eddy simulation, is revised. The principle of material frame indifference and the principle of tu rbulent frame indifference proposed b y Hutter and Joenk imposes that both constitutive equations and turbulent closure rela tions must respect both the require ment of form invariance, and the requirement of frame independence. In this paper, a new rule for the form alization of turbulent closure relations, is proposed. The generalized SGS turbulent stress tensor is related exclusively to the generalized SGS turbulent kinetic en ergy, which is calculated by means of its balance equation, a nd the modified Leonard tensor.
\end{abstract}

Key-Words: - Large eddy simulation, Noll's axioms, frame indifference, form invariance, frame dependence, turbulent closure relations

Received: August 16, 2019. Revised: February 7, 2020. Accepted: April 1, 2020. Published: April 30, 2020.

\section{The formulation of the Principle of Material Frame Indifference and the Principle of Turbulent Frame Indifference}

In the framework of or dinary fluid dynamics, the turbulence models could be $\mathrm{i}$ nterpreted as constitutive equations, which are necessary to close the equations of motion. The constitutive equations represent, in an idealized form, the behaviour of the materials and, consequently, the y must fulfil the principle of material frame indifference [1].

In order to make explicit the relation between the Noll formulation of the principle of material frame indifference and the form ulation of the turbulen $t$ closure relations, in $\mathrm{t}$ his section: the Noll formulation of the principle of material frame indifference is shown; the confusion, produced by this formulation, is underlined (it does not emphasize the difference between Euclidean form invariance and frame independence of an equation); the distinction between $\mathrm{E}$ uclidean form invariance and frame in dependence of a constitutive equation or physical law is explained; the Hutter and Joenk [2] formulation of the principle of turbulent frame indifference, that is the eq uivalent in turbulence of the principle of material frame indifference, i s expressed.

Considering an i nertial frame, in which a material point has coordinate $x_{i}$ at time $t$, and a noninertial frame, in which the same point has coordinate $x_{i}^{*}$ at time $t^{*}$, the most general law which governs the transformations of the coo rdinates and the time expressed in the two frames is that given by the Euclidean transformations

$$
x_{i}=Q_{i j}(t) x_{j}^{*}+b_{i}(t) \quad t=t^{*}+a
$$

where $Q_{i j}(t)$ are the co mponents of a tim edependent proper orthogonal tensor, $b_{i}(t)$ is the time-dependent distance between the origins of the two frames and $a$ is any constant.

It is comm on knowledge that tensors of rank $n$ $(n=0,1,2)$ are said to be obje ctive, if the components transform according to:

$$
\begin{array}{cc}
S=S^{*} & \text { objective scalar } \\
V_{i}=Q_{i j} V_{j}^{*} & \text { objective vector } \\
A_{i j}=Q_{i m} Q_{j n} A_{m n}^{*} & \text { objective tensor }
\end{array}
$$

A constitutive relation can be expressed in the form

$$
T(\vec{\chi}, t)=F\left(\vec{\chi}^{t}, t\right)
$$

where $\vec{\chi}^{t}$ is the history of the motion of the body $B$ up to the time $t$ and $T(\vec{x}, t)$ is the stress tensor. The principle of material frame indifference is based on the consideration that the material properties must be independent of the choice of frame. In other words, this basic working principle of continuum mechanics requires the cons titutive equations to be the same for observers in inertial sy stems and in 
non-inertial ones. Since constitutive equations are designed to express idealized material properties, the Noll for mulation of the principle of $m$ aterial frame indifference requires they shall be fra me independent. That is, if the constitutive relation (2) is satisfied by the dynamic proces $\mathrm{s}(\vec{\chi}, T)$, it is satisfied by every equivalent process $\left(\vec{\chi}^{*}, T^{*}\right)$ that is represented in a non-iner tial frame of reference. Formally, the constitutive mapping $F$ in (2) m ust satisfy the identity

$$
T\left(\vec{\chi}^{*}, t^{*}\right)^{*}=F\left(\vec{\chi}^{*}, t^{*}\right)
$$

For all $T^{*}, \vec{\chi}^{*}$ and $t^{*}$ that may be obtained from $T$, $\vec{\chi}$ and $t$ by Euclidean transformations of the frame expressed by relations (1) and (2) [1 ]. The abovementioned Noll formulation of the principle of material frame indifference produces a confusion because it does not e mphasize the difference between two distinct requirements on $t$ he constitutive equations: form invariance under Euclidean transformations of the fr ame; frame independence.

1) The requirement of Euclidean for $m$ invariance implies the $f$ ormal expression of the constitutive equations in a non-inertial frame of reference be equal to the formal expression of the constitutive equation in an inertial fram e of reference: that is, a constitutive equation is Euclidean form invariant if it does not $\mathrm{m}$ odify its formal expression under Euclidean transformation of the fram e and, co nsequently, it is constructed only with objective tenso rs. In other words, each observer uses the constitutive equations in the same functional form, but the quantities appearing in them may have di fferent values due to the used fram e, i.e., the values of the qua ntities appearing in them may be frame dependent.

2) The requirement of frame independence of a constitutive equation $i$ mplies the values of the quantities, appearing in it, be $\mathrm{i}$ ndependent of translational and angular velocity of the frame. It is possible to em phasize the difference between the

Euclidean form invariance and the fra me independence by underlining the existence of tensors that are objective but dependent on the translational and angular velocity of the frame.

For example, let $W_{i j}$ and $W_{i j}^{*}$ be, respect ively, the representations, in an inertial and non-i nertial frame, of th e antisymmetric part of the velocity gradient. Let $W_{i j}^{\Omega *}$ be the repr esentation, in a noninertial frame, of the absolute vorticity tensor, given by the following expression:

$$
W_{i j}^{\Omega *}=W_{i j}^{*}+Q_{k i} Q_{k j}
$$

The law of transformation between th representations of this ten sor in the different fra mes of reference is given by:

$$
W_{i j}^{\Omega *}=Q_{i m} Q_{j n} W_{m n}^{\Omega *}
$$

The absolute vorticity tensor $W_{i j}^{\Omega}$ is an objective tensor, since its represen tations in the different frames transform according to equation (2), but is frame dependent since its representations depend on the frame by means of the term $Q_{k m} \dot{Q}_{k n}$, associated with the angular velocit $y$ of the non-i nertial frame [2-3].

A constitutive equation, or a phy sical law, in order to be form invariant under the $m$ ost general class of transfor mations of the fram e (Euclidean transformations), must be expressed i $n$ terms of objective tensors. A constitutive equation, or a physical law, is able to fulfil the principle of material frame indifference if: it is form invariant (under Euclidean transformation of the frame); it is frame independent, i.e. it is expressed exclusively in terms of objective tensors that are in dependent of the translational and angular velocity of the frame.

The principle of turbulent frame indifference [2] is the equivalent in turbul ence of the principle of material frame indifference. The principle of turbulent frame indifference imposes that turbulent closure relation: must be form invariant (or rather, must be expressed in ter $\mathrm{ms}$ of objective tensors); must be frame independent (or rath er, must be expressed in terms of objective tens ors that are independent of the angular and translational velocity of the frame).

\section{Turbulent Balance Equations in Large Eddy Simulation}

It must be emphasized that Euclidean form invariance and frame independence are two distinct matters.

The generalised SGS turbulent stress tensor is expressed by the equation

$$
\tau_{i j}=\overline{u_{i} u_{j}}-\bar{u}_{i} \bar{u}_{j}
$$

where $u_{i}$ is the $i-t h$ component of the instantaneous velocity and the overbar represents the application of spatial filter.

Following the procedure shown in [4], in order to show the characteristi $\mathrm{c}$ of the generalised SGS turbulent stress tensor an $\mathrm{d}$ in order to define the 
modalities of form ulation of the turbulent closure relations, later on we present:

- the objectivity and the frame independence of the abovementioned tensor;

- the Euclidean form invariance and frame dependence of the generalised SGS turbulent stress tensor transport equation;

- the Euclidean form invariance and frame independence of the generalised SGS turbulent kinetic energy transport equation.

\subsection{The generalized SGS turbulent stress tensor}

The time derivative of (1) gives

$$
u_{i}=Q_{i j}(t) u_{j}^{*}+\dot{Q}_{i j}(t) x_{j}^{*}+\dot{b}_{l}(t)
$$

Applying a spatial filter to (8) gives

$$
\bar{u}_{i}=Q_{i j}(t) \bar{u}_{j}^{*}+\dot{Q}_{l \jmath}(t) x_{j}^{*}+\dot{b}_{l}(t)
$$

By introducing (8) and ( 9) into (7), the relation between the expressions of the generalised SGS turbulent stress tensor in two Euclidean frames is obtained,

$$
\tau_{i j}=Q_{i l} Q_{j m}\left(\overline{u_{l}^{*} u_{m}^{*}}-\bar{u}_{l}^{*} \bar{u}_{m}^{*}\right)=Q_{i l} Q_{j m} \tau_{l m}^{*}
$$

Equation (10) shows that the generalised SGS turbulent stress tensor i $\mathrm{s}$ objective and fra me independent. Consequently, all of the turbulen $t$ closure relations for $\mathrm{t}$ he generalised SGS turbulen $\mathrm{t}$ stress tensor must be: form invariant under Euclidean transformations of the frame; independent of the transl ational and angular velocity of $t$ he frame.

\subsection{Transport equation of the generalised SGS turbulent stress tensor}

The transport equation of the generalized SGS turbulent stress tensor is:

$$
\begin{gathered}
\frac{D \tau\left(u_{k}, u_{l}\right)}{D t}=-\frac{\partial \tau\left(u_{k}, u_{l}, u_{p}\right)}{\partial x_{p}}-\tau\left(u_{p}, u_{k}\right) \frac{\partial \bar{u}_{l}}{\partial x_{p}}- \\
\tau\left(u_{p}, u_{l}\right) \frac{\partial \bar{u}_{k}}{\partial x_{p}}-\tau\left(u_{k}, \frac{\partial p}{\partial x_{l}}\right)-\tau\left(u_{l}, \frac{\partial p}{\partial x_{k}}\right)+ \\
v \tau\left(u_{k}, \frac{\partial^{2} u_{l}}{\partial x_{p} \partial x_{p}}\right)+v \tau\left(u_{l}, \frac{\partial^{2} u_{k}}{\partial x_{p} \partial x_{p}}\right)
\end{gathered}
$$

The symbols $\tau(f ; g)$ and $\tau(f ; g ; h)$ represent the generalized second and third-order central moments [5] related to the generic quantities $f, g$ and $h$.

By following the procedure shown in [4], by introducing (1), (8), (9) and (10) i $\mathrm{n} \mathrm{(11),} \mathrm{t} \mathrm{he}$ representation in a non-inertial fra me of the generalized SGS turbule nt stress tensor transport equation is:

$$
\begin{gathered}
Q_{k m} Q_{l n}\left[\frac{D \tau\left(u_{m}^{*}, u_{n}^{*}\right)}{D t *}+Q_{r n} \dot{Q}_{r p} \tau\left(u_{p}^{*}, u_{m}^{*}\right)+\right. \\
\left.Q_{r m} \dot{Q}_{r p} \tau\left(u_{p}^{*}, u_{n}^{*}\right)\right]= \\
Q_{k m} Q_{l n}\left[-\frac{\partial \tau\left(u_{p}^{*}, u_{m}^{*}, u_{n}^{*}\right)}{\partial x^{*} p}-\tau\left(u_{p}^{*}, u_{m}^{*}\right) \frac{\partial \bar{u}_{n}^{*}}{\partial x_{p}^{*}}-\right. \\
Q_{r n} \dot{Q}_{r p} \tau\left(u_{p}^{*}, u_{m}^{*}\right)-\tau\left(u_{p}^{*}, u_{n}^{*}\right) \frac{\partial \bar{u}_{m}^{*}}{\partial x_{p}^{*}}- \\
Q_{r m} \dot{Q}_{r p} \tau\left(u_{p}^{*}, u_{n}^{*}\right)-\tau\left(u_{m}^{*}, \frac{\partial^{*} p}{\partial x_{n}}\right)- \\
\tau\left(u_{n}^{*}, \frac{\partial^{*} p}{\partial x_{n}}\right)+v^{*} \tau\left(u_{m}^{*}, \frac{\partial^{2} u_{n}^{*}}{\partial x_{p}^{*} \partial x_{p}^{*}}\right)+ \\
\left.v^{*} \tau\left(u_{n}^{*}, \frac{\partial^{2} u_{m}^{*}}{\partial x_{p}^{*} \partial x_{p}^{*}}\right)\right]
\end{gathered}
$$

By using the expression $W_{i j}^{\Omega *}$ of the absolute vorticity tensor defined in (6) and the objective time derivative introduced by Weis and Hutter [ 3], equation (12) reads

$$
\begin{gathered}
\frac{\widetilde{D} \tau\left(u_{m}^{*}, u_{n}^{*}\right)}{D t^{*}}=-\frac{\partial \tau\left(u_{p}^{*}, u_{m}^{*}, u_{n}^{*}\right)}{\partial x_{p}^{*}}- \\
\tau\left(u_{m}^{*}, u_{p}^{*}\right)\left(\bar{S}_{p n}^{*}-\bar{W}_{p n}^{* \Omega}\right)-\tau\left(u_{p}^{*}, u_{n}^{*}\right)\left(\bar{S}_{m p}^{*}-\right. \\
\left.\bar{W}_{m p}^{* \Omega}\right)-\tau\left(u_{m}^{*}, \frac{\partial p^{*}}{\partial x_{n}}\right)-\tau\left(u_{n}^{*}, \frac{\partial p^{*}}{\partial x_{n}}\right)+ \\
v^{*} \tau\left(u_{m}^{*}, \frac{\partial^{2} u_{n}^{*}}{\partial x_{p}^{*} \partial x_{p}^{*}}\right)+v^{*} \tau\left(u_{n}^{*}, \frac{\partial^{2} u_{m}^{*}}{\partial x_{p}^{*} \partial x_{p}^{*}}\right)
\end{gathered}
$$

Since the absolute vortici ty tensor $W_{i j}^{\Omega *}$ and the time derivative $\widetilde{D} \tau\left(u_{m}^{*}, u_{n}^{*}\right) / D t^{*}$ are both objective tensors, equation (13) is expressed exclusively in terms of objective tensors.

From this consideration and for the assumption that an equation is form invariant if it is expressed only in terms of objective tensors, it results that the transport equation of the generalised SGS turbulent stress tensor is form invariant under a Euclidean transformation of the fra me but remains fra me dependent through the apparition of $W_{i j}^{\Omega *}$.

From the previous considerations, it can be deduced that the principle of turbulent fra me indifference proposed by Hutter and Joenk [2] couldn't be applied to th e transport equation of the generalized SGS turbulent stress tensor.

\subsection{Transport equation of the generalised SGS turbulent kinetic energy}

The generalized SGS turbulent ki netic energy $E$ is defined as half the trace of the SGS turbulent stress tensor and is, as can be easily demonstrated, an objective scalar. The ge neralized SGS turbulent kinetic energy transport equation is [4]: 


$$
\begin{gathered}
\frac{1}{2} \frac{D \tau\left(u_{k}, u_{k}\right)}{D t}=-\frac{1}{2} \frac{\partial \tau\left(u_{k}, u_{k}, u_{p}\right)}{\partial x_{p}}- \\
\tau\left(u_{m}, u_{k}\right) \frac{\partial \bar{u}_{k}}{\partial x_{m}}-\tau\left(u_{k}, \frac{\partial p}{\partial x_{k}}\right)+ \\
v \tau\left(u_{k}, \frac{\partial^{2} u_{k}}{\partial x_{p} \partial x_{p}}\right)
\end{gathered}
$$

which is equal to

$$
\begin{gathered}
\frac{D E}{D t}=-\frac{1}{2} \frac{\partial \tau\left(u_{k}, u_{k}, u_{m}\right)}{\partial x_{m}}-\tau\left(u_{m}, u_{k}\right) \frac{\partial \bar{u}_{k}}{\partial x_{m}}- \\
\frac{\partial \tau\left(p, u_{m}\right)}{\partial x_{m}}+v \frac{\partial^{2} E}{\partial x_{m} \partial x_{m}}-v \tau\left(\frac{\partial u_{k}}{\partial x_{m}}, \frac{\partial u_{k}}{\partial x_{m}}\right)
\end{gathered}
$$

By introducing (1), (8), (9) and (10) in (15), the representation in a non-inertial fra me of the generalized SGS turbulent kinetic energy transport equation is:

$$
\begin{gathered}
\frac{D E^{*}}{D t^{*}}=-\frac{1}{2} \frac{\partial \tau\left(u_{m}^{*}, u_{m}^{*}, u_{p}^{*}\right)}{\partial x_{p}^{*}}-\tau\left(u_{p}^{*}, u_{m}^{*}\right) \frac{\partial \bar{u}^{*} m}{\partial x_{p}^{*}}- \\
\frac{\partial \tau\left(p^{*}, u_{k}^{*}\right)}{\partial x_{k}^{*}}+v^{*} \frac{\partial^{2} E^{*}}{\partial x_{p}^{*} \partial x_{p}^{*}}-v^{*} \tau\left(\frac{\partial u_{p}^{*}}{\partial u_{n}^{*}}, \frac{\partial u_{p}^{*}}{\partial u_{n}^{*}}\right)
\end{gathered}
$$

From the com parison between equations (15) and (16), it can be deduced that the transport equation of the generalized SGS turbulent kinetic energy is form invariant and frame independent, in so much that each of th $\mathrm{e}$ terms that appear in it are representations, in inertial and non-inertial frames, of objective tensors that are indepen dent of the angular and translational velocity of the frame.

\section{A New Rule of Turbulent Closure Relations}

In the pre vious section, the Euclidean for $\mathrm{m}$ invariance and the fra me dependence of the generalised SGS turbule nt stress tensor transport equation, has been demonstrated. Many authors repute that all of the turbulent closure relations must fulfil the principle of turbulent frame indifference in the formulation proposed by Hutter and Joenk [2]. A contradiction arises from the abo vementioned imposition: the generalised SGS tur bulent stress tensor transport equation could not be used in the turbulent closure relations, since it does n't fulfil the principle of turbulent frame indifference. Must all of the turbulent closure relations fulfil the principle of turbulent frame indifference? In other words, if $y=$ $c(x)$ is a turbul ent closure relation, does this relation have to be frame independent? No, it does not need to.

It is usually assumed that material laws do not depend on the rotation of the sy stem. This means that in every system the material should show the same behaviour. This is quite a good assu mption as long as the r elaxation time of the $\mathrm{m}$ aterial is large compared with the typical time scale of the flow.

The turbulent phenomena are not associated to the properties of the materials: consequentl $y$, turbulent closure relations do not re present the material behaviour. In such flows the characteristic turbulent time scale can be co mparable with the typical time scale of the flow, i mplying that the rotation of the sy stem can influence the turbulent closure functionals. This means that objective tensors, which depend on the rotation of the reference frame, may enter such functional relations. Constitutive relations of any turbulence theor y need not satisfy the princi ple of turb ulent frame indifference.

Turbulent closure relations must always be form invariant but $\mathrm{m}$ ust not necessarily be frame independent. In other wo rds, not all the turbulent closure relations $\mathrm{m}$ ust fulfil the principle of turbulent frame indifference. A new rule of turbulent closure relations can be formulated:

"In a turbulent closure relation, the modelled expressions of an unknown objective ten sor must be formulated in term $\mathrm{s}$ of objective tensors, allowing the closure relations to fulfil the requirem ent of Euclidean form invariance, and must retain the same dependence on the angular velocity of the frame of the unknown tensor".

\section{Closure Relations}

In depth-averaged motion equations models [6-7] and in models based on 3D Navier-Stokes equations [8-10], the $t$ urbulent stress tensor is $r$ elated to the strain rate tensor, which is Reynolds-averaged. In the context of LES $m$ odels, the generalized SGS turbulent stress tensor, $\tau_{i j}$, is related to the resolved tensors. The generalised SGS turbulent stress tensor, $\tau_{i j}$, can be split into three tensors:

$$
\tau_{i j}=\overline{u_{\imath} \bar{u}_{\jmath}}-\bar{u}_{i} \bar{u}_{j}=L_{i j}^{m}+C_{i j}^{m}+R_{i j}^{m}
$$

where $u_{i}$ is the $i$-th component of the instantaneous velocity, the overbar rep resents the a pplication of the grid filtering operator, $L_{i j}^{m}, C_{i j}^{m}$ and $R_{i j}^{m}$ are the so-called modified Leonard tensor, th e modified cross tensor and $\mathrm{t}$ he modified Re ynolds tensor, respectively; $u_{i}^{\prime}$ is the $i$-th component of the fluctuating velocity, $u_{i}^{\prime}=u_{i}-\bar{u}_{i}$.

Starting from (17), by adopti ng the scale similarity assumption, by simple mathematical calculations, a closure rel ation is reac hed for the generalized SGS turbule nt stress tensor, in whic $h$ there are no coefficients to be calibrated or to be 
calculated dynamically, and which is given by the following relation:

$$
\tau_{i j}=2 E \frac{L_{i j}^{m}}{L_{k k}^{m}}
$$

where $E=\frac{\tau_{k k}}{2}$. See [4] for the details.

The generalised SGS turbulent stress tensor is related exclusively to the generalised SGS turbulent kinetic energy and the modified Leonard tensor that are, respectively, a zero o rder and a second order objective tensor that a re independent of the translational and angular velocit $y$ of the frame. Consequently, the closure relation (18) for the generalised SGS turbulent stress tensor : takes into account the anisotropy of the turbulence; rem oves any balance assumption between the production and dissipation of SGS turb ulent kinetic e nergy; does not use any closure coefficient calculated by means of a dy namic procedure; respect $s$ the new rule of turbulent closure relations, proposed in section 3 .

The generalised turbulent kinetic energ y $E$ is calculated by solving its transport equation (15) . The proposed modelled form of Equation (15) is:

$$
\begin{gathered}
\frac{D E}{D t}=\frac{\partial}{\partial x_{k}}\left(D \sqrt{E} \bar{\Delta} \frac{\partial E}{\partial x_{k}}\right)-\left(\frac{2 E}{L_{q q}^{m}}\right) L_{m k}^{m} \frac{\partial \bar{u}_{k}}{\partial x_{m}}+ \\
v \frac{\partial^{2} E}{\partial x_{m} \partial x_{m}}-\frac{C_{*} E^{3 / 2}}{\bar{\Delta}}
\end{gathered}
$$

where the $1^{\text {st }}$ and $3^{\text {rd }}$ terms on the right-hand side of the exact balance equation of $E$ (15) are modelled by the $1^{\text {st }}$ term of equation (19); the last term of the right-hand side of equation (15), whi ch represents the viscous dissipation of the tur bulent kinetic energy, is modelled b y the last term on the righthand side of equation (19); the values of the coefficients $C_{*}$ and $D$ are eval uated by means of a dynamic procedure.

\section{Results and discussion}

Turbulent channel flows (between two flat parallel plates placed at a distance of $2 L$ ) are simulated with the model that uses the $\mathrm{p}$ resented closure relation, hereinafter called TEM model, at friction-velocitybased Reynolds number $R e^{*}$ equal to 2340 . The numerical results obtained with the TEM model are compared with experimental data [11].

Figure 1a shows the profile of the time-averaged streamwise velocity component for a channel flow at $R e^{*}=2340$ obtained with the TEM model, compared with the profile of the analogous velocity component measured experimentally [ 11]. The agreement between the two velocity profiles is very good.
Figure $1 \mathrm{~b}$ compares the profile of the component $\left\{u_{1}^{\prime} u_{3}^{\prime}\right\}$ of the Re ynolds stress tensor (where the subscripts 1 and 3 denote, respecti vely, the streamwise and wall-nor mal directions), calculated with the TEM model, with the profile of the similar component of the Rey nolds stress ten sor obtained from experimental measurements [11], for a channel flow at $R e^{*}=2340$. Figure $1 \mathrm{~b}$ shows that at the TEM model provides a profile of $t$ he component $\left\{u_{1}^{\prime} u_{3}^{\prime}\right\}$ in agreement with the one obtained from the experimental measurements.
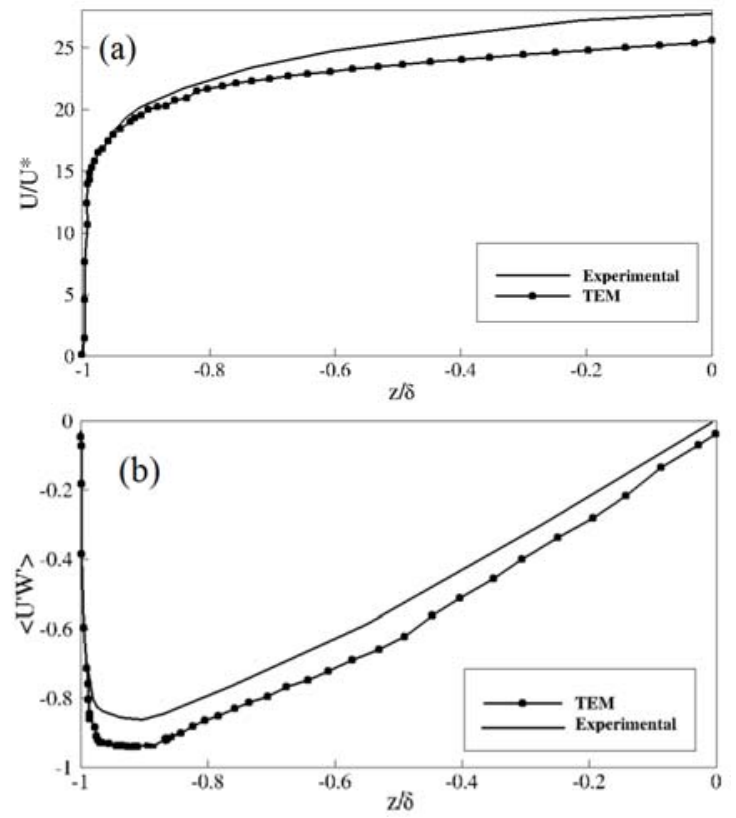

Figure 1. Comparison between experimental measurements and LES $r$ esults obtained with the TEM model. Channel flow, $R e^{*}=2340$. (a): Timeaveraged streamwise velocities. (b) Reynolds stress $\left\{u_{1}^{\prime} u_{3}^{\prime}\right\}$.

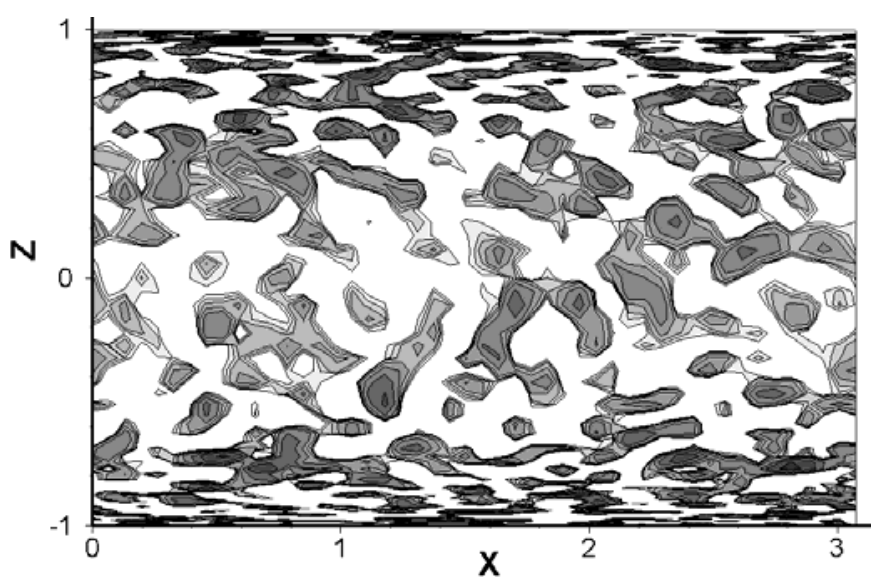

Figure 2. Vortex identification with $\lambda_{2}$ method, $x-z$ plane.

In figure 2 the near-wall vortex structures (inside the turbulent boundary layer) are clear ly identified by the $\lambda_{2}$ method of Joeng \& Hussain [ 12]: the 
dimensions of the spatial discretization steps allow the optimal simulation of the ab ovementioned vortex structures that govern the transport, the production and the dissipation of th e turbulent kinetic energy. See also [13] and [14].

\section{Conclusion}

The relation between Noll's form ulation of the principle of material frame indifference and the principle of turbulent frame indifferen ce, has been revised. The definition of a new Rule of Turbulent Closure Relations has been proposed. The aforementioned rule of Turbulent Closure Relations has been expressed in the following form : "In a turbulent closure relation, the modelled expressions of an unknown objective tensor must be formulated in terms of objective tensors, allowing the closure relations to fulfil the requirem ent of Euclidean form invariance, and must retain the same dependence on the angular velocity of the frame of the unk nown tensor". The generalized SGS turbulent stress tensor is related exclusively $t \quad o$ the generalized SGS turbulent kinetic energy, which has be en calculated by means of its balance equation, and the modified Leonard tensor.

\section{References:}

[1] Truesdell C. 1993 A first course in rational continuum mechanics Academ ic Press (New York)

[2] Hutter K., Joenk K. 2004 Continu um Methods of Physical Modelling Springer (Berlin)

[3] Weis J., Hutter K. On Euclidean invariance of algebraic Reynolds stress models in turbulence Journal of Fluid Mechanics, Vol. 476, 2003, pp. 63-68.

[4] Gallerano F., Pasero E., Cannata G. A dynamic two-equation Sub Grid Scale model. Continuum Mechanics and Thermodynamics, Vol. 17, No. 2, 2005, pp. 101-123.

[5] Germano M. Turbule nce: the filtering approach. Journal of Fluid Mechanics, Vol. 238, 1992, pp. 323-336

[6] Cannata G., Petrelli C., Barsi L., Fratello F., Gallerano F. A dam -break flood si mulation model in curvilinear coordinates. WSEAS Transactions on Fluid Mechanics, Vol. 13, 2018, pp. 60-70.

[7] Cannata G., Barsi L., Pe trelli C., Gallerano F. Numerical investigation of wave fields and currents in a coastal engineering case study.
WSEAS Transaction on Fluid Mechanics, Vol. 13, 2018, pp. 87-94.

[8] Cannata G., Petrelli C., Barsi L., Camilli F., Gallerano F. 3D free surface flow simulations based on the integral form of the equations of motion. WSEAS Transaction on Fluid Mechanics, Vol. 12, 2017, pp. 166-175.

[9] Cannata G., Gallerano F., Palleschi F., Petrelli C., Barsi L. Three-dimensional n umerical simulation of the velocity fields induced by submerged breakwaters. International Journal of Mechanic, Vol. 13, 2019, pp. 1-14.

[10] Cannata G., Petrelli C., Barsi L., Gallerano F., Numerical integration of the contr avariant integral form of the Navier-Stokes equations in time-dependent curvilinear coordinate sy stem for three-dimensional $f$ ree surface flows, Continuum Mechanics and Thermodynamics, Vol. 31, No. 2, 2019, pp.491-519.

[11] Comte-Bellott G AGARD Advisory Report, 343

[12] Joeng J., Hussain F. On the identificati on of a vortex. Journal of Fluid Mechanics, Vol. 285, 1995, pp. 69-94.

[13] Tadashi Watanabe, Nu merical Simulation of Droplet Combustion using Volum e-of-Fluid Method, WSEAS Transactions on Heat and Mass Transfer, Volume 14, 2019, pp. 38-44

[14] Kun Dou, Qing Liu, Lingtao Meng, Study of Fluid Flow, Heat Transfer and Surface Wave Vibration Behaviour for Steel in Continuous Casting Process Using Mathematical Modelling and Physical Modelling, WSEAS Transactions on Heat and Mass Transfer, Volume 14, 2019, pp. 153-157 